\title{
Levantamento da intensidade da alternariose e da podridão negra em cultivos orgânicos de brássicas em Pernambuco e Santa Catarina
}

\author{
Luiz A M Peruch ${ }^{1}$; Sami J Michereff"; Issac B Araújoº ${ }^{2}$ \\ ${ }^{1}$ EPAGRI-Urussanga; 88840-000 Urussanga-SC; ${ }^{2}$ UFRPE, 52171-900 Recife-PE; *Bolsista CNPq; E-mail: lamperuch@epagri.rct-sc.br
}

\begin{abstract}
RESUMO
O objetivo deste trabalho foi avaliar a intensidade da alternariose, causada por Alternaria brassicicola e/ou Alternaria brassicae, e da podridão negra, causada por Xanthomonas campestris pv. campestris, em cultivos orgânicos de brássicas em Pernambuco e Santa Catarina. Os levantamentos foram realizados no período de novembro de 2001 a fevereiro de 2002, num total de 103 cultivos orgânicos de várias espécies de brássicas. Foram registradas elevadas prevalências das doenças nos estados, com exceção em couve-chinesa em Santa Catarina. A prevalência da alternariose foi $100 \%$ nos cultivos de brócolis em Pernambuco, bem como em couve-flor nos dois estados, enquanto a podridão negra atingiu esse nível nos cultivos de brócolis e couve-flor em Santa Catarina. Na média das diferentes espécies de brássicas, as doenças foram mais prevalentes em Pernambuco que Santa Catarina. Entretanto, as médias de severidade de cada doença no conjunto das brássicas não foram diferentes entre os estados, embora as condições climáticas tenham sido nitidamente distintas. A severidade da alternariose variou entre as espécies de brássicas somente em Pernambuco, com a menor severidade registrada em couve-manteiga. Em relação à podridão negra, apenas em Santa Catarina houve diferença na severidade entre as brássicas, sendo registrados os menores níveis em couve-chinesa. Não foram constatadas correlações significativas entre os níveis de severidade da alternariose e da podridão negra, bem como da severidade destas com o número total de plantas e a idade das plantas nos cultivos.
\end{abstract}

Palavras-chave: Alternaria brassicicola, Alternaria brassicae, Xanthomonas campestris pv. campestris, severidade, prevalência.

\begin{abstract}
Survey of the intensity of Alternaria black spot and black rot on brassica species under organic farming systems in Pernambuco and Santa Catarina states, Brazil

The objective of this research was to evaluate the intensity of Alternaria black spot, caused by Alternaria brassicicola and/or Alternaria brassicae, and black rot, caused by Xanthomonas campestris pv. campestris in organic cultivation of brassicas in Pernambuco and Santa Catarina. The survey was carried aut from November 2001 to February 2002, in 103 fields under organic farming systems with different brassicas. High prevalence of the diseases was registered in both states, except on Chinese cabbage in Santa Catarina. Prevalence of Alternaria black spot was $100 \%$ on broccoli fields in Pernambuco, as well as on cauliflower in both states, while the black rot reached that level on broccoli and cauliflower fields in Santa Catarina. On the average of the different brassica species, the diseases were more prevalent in Pernambuco than in Santa Catarina. However, when the severity averages of each disease were considered, no significant differences were observed between the two states, although the climatic conditions were highly different. The Alternaria black spot severity varied among the brassica species in Pernambuco, being lower on kale. In Santa Catarina no significant differences were observed among the brassicas species. In relation to the black rot, only in Santa Catarina was there a difference in the disease severity, with the lowest level on Chinese cabbage. No significant correlations were observed either between severity levels of Alternaria black spot and black rot, neither between disease severity and total number of plants or plant age.
\end{abstract}

Keywords: Alternaria brassicicola, Alternaria brassicae, Xanthomonas campestris pv. campestris, severity, prevalence.

(Recebido para publicação em 12 de dezembro de 2005; aceito em 1 de novembro de 2006)

$\mathrm{O}$ cultivo de brássicas tem destacada importância na olericultura orgânica brasileira, devido ao grande volume de produção, ao retorno econômico propiciado e ao valor nutricional das culturas. Mesmo adaptadas às condições edafo-climáticas, a produção de brássicas pode ser limitada pela ocorrência de doenças, dentre as quais se destacam a alternariose, causada por Alternaria brassicicola (Schwn.) Wilt. e/ou Alternaria brassicae (Berk.) Sacc., e a podridão negra, causada por Xanthomonas campestris pv. campestris (Pammel) Dowson, e que provocam consideráveis reduções na produtividade e na qualida- de dos produtos (Maringoni, 1997; Williams, 1980). Essas doenças se desenvolvem em qualquer estádio de desenvolvimento das plantas, são transmitidas por sementes e infectam as principais brássicas de interesse econômico, sendo que em períodos úmidos as culturas de brócolis (Brassica oleracea var. italica L.), couve-flor (Brassica oleracea var. botrytis L.) e repolho (Brassica oleracea var. capitata L.) podem ter a sua produção reduzida em mais de 50\% (Ramsey \& Smith, 1961; Verma \& Saharan, 1994; Williams, 1980).

A alternariose causa danos maiores quando ocorre na fase de sementeira, onde provoca necrose dos cotilédones, hipocótilo e tombamento, o que acarreta a destruição das plântulas ou inviabiliza o transplantio. Em plantas adultas, os sintomas ocorrem inicialmente nas folhas mais velhas, caracterizados por lesões pequenas e necróticas e, posteriormente, infecta folhas mais jovens, apresentando lesões circulares, concêntricas e com halo clorótico. A podridão negra caracteriza-se por lesões amarelas em forma de "V", com o vértice voltado para o centro da folha. Com o desenvolvimento da doença, as folhas tornam-se amarelas e podem apresentar necrose. Em alguns casos, observa- 
se subdesenvolvimento, murcha, queda prematura de folhas e apodrecimento das plantas afetadas (Maringoni, 1997).

O desenvolvimento de sistemas orgânicos de produção, alternativos à utilização intensiva de defensivos químicos, insumos inorgânicos e práticas com baixa sustentabilidade, vêm despertando cada vez mais interesse no cenário agrícola mundial, embora exista pouco conhecimento institucional sobre esses sistemas, incluindo os aspectos relacionados às doenças de plantas (van Bruggen, 2001). Nesse contexto, apesar do cultivo orgânico de hortaliças estar em franca expansão no Brasil, existe grande deficiência de informações sobre o manejo de doenças nesse sistema de produção (Rodrigues et al., 2004). Em relação às brássicas, a expansão da produção orgânica não foi acompanhada de estudos sobre a intensidade das doenças, tendo em vista que inexistem levantamentos da severidade da alternariose e da podridão negra em cultivos orgânicos nas condições brasileiras.

Levantamentos fitopatológicos têm como objetivos fornecer informações sobre a importância relativa das doenças, monitorar flutuações nas suas intensidades e verificar a eficiência e aceitação de práticas recomendadas de controle (King, 1980), constituindo-se, desta forma, num importante instrumento para o desenvolvimento de programas de manejo integrado de doenças (Campbell \& Madden, 1990). Um aspecto pouco explorado nesses levantamentos é a comparação da intensidade das doenças entre regiões produtoras localizadas em diferentes partes do país e com características climáticas distintas. Portanto, o objetivo deste trabalho foi realizar o levantamento da intensidade da alternariose e da podridão negra em cultivos orgânicos de brássicas nos estados de Pernambuco e Santa Catarina.

\section{MATERIAL E MÉTODOS}

Nos períodos de novembro a dezembro de 2001 e de janeiro a fevereiro de 2002, foram realizados levantamentos da intensidade da alternariose e da podridão negra em plantios orgânicos de brássicas nos estados de Pernambuco e
Santa Catarina, respectivamente. Os cultivos orgânicos atendiam as exigências da Instrução Normativa ${ }^{\circ} 007$, de 19/05/1999, do Ministério da Agricultura, Pecuária e Abastecimento (MAPA), que dispõe sobre a produção de produtos orgânicos vegetais e animais. Em Pernambuco foram amostrados 20 cultivos de brócolis, seis de couve-chinesa (Brassica pekinnensis L.), nove de couve-flor, 19 de couvemanteiga (Brassica oleracea L. var. acephala DC.) e 10 de repolho, enquanto em Santa Catarina foram amostrados 15 cultivos de brócolis, três de couvechinesa, dois de couve-flor, 14 de couve-manteiga e cinco de repolho.

Utilizando-se a técnica de caminhamento em "W", em cada cultivo foram selecionadas 50 plantas ao acaso, distribuídas em canteiros. A severidade foi determinada em cinco folhas basais de cada planta, pela porcentagem de área foliar lesionada, com o auxílio de escalas diagramáticas apresentando níveis de 0 a $50 \%$ para alternariose (Conn et al., 1990) e de 0 a $32 \%$ para podridão negra (Azevêdo $e t$ $a l ., 2000)$. A prevalência regional de cada doença foi determinada pela porcentagem de áreas com a doença em relação ao total de áreas amostradas (Campbell \& Madden, 1990).

Para verificação das espécies predominantes de Alternaria causadoras da alternariose, em todos os plantios com a ocorrência da doença foram efetuadas coletas de folhas com sintomas. Em laboratório, fragmentos de lesões foram retirados das folhas e mantidos em câmara úmida por 72 horas. Posteriormente, foram preparadas lâminas e observadas ao microscópio ótico, sendo efetuadas as identificações (Verma \& Saharan, 1994).

Informações adicionais sobre localização geográfica, cultivar, número de plantas e idade das plantas, foram obtidas em cada cultivo. Dados diários de temperatura e precipitação pluviométrica foram obtidos nas regiões amostradas, em estações meteorológicas.

Foram efetuadas comparações das médias de severidade de cada doença em relação às espécies de brássicas e entre os estados. As culturas de brócolis e couve-manteiga, que tiveram pelo menos 14 cultivos amostrados em cada estado, foram separadas em classes considerando local de cultivo, cultivar, número de plantas e idade das plantas, cujos níveis de severidade das doenças foram comparados. Todas as comparações de médias foram realizadas com testes não-paramétricos ao nível de 5\% de probabilidade, sendo utilizado o teste de Wilcoxon quando comparadas duas médias e o teste de Kruskal-Wallis quando comparadas mais de duas médias. Foi também efetuada a análise de correlação de Pearson, ao nível de 5\% de probabilidade, entre severidades das doenças, números de plantas e idades das plantas.

\section{RESULTADOS E DISCUSSÃO}

Foram registradas elevadas prevalências da alternariose e da podridão negra em cultivos orgânicos de brássicas nos estados de Pernambuco e Santa Catarina, com exceção em couve-chinesa no último estado (Tabela 1). A prevalência da alternariose foi $100 \%$ nos cultivos de brócolis em Pernambuco, bem como em couve-flor nos dois estados. A prevalência da podridão negra atingiu $100 \%$ nos cultivos de brócolis e couve-flor, ambos em Santa Catarina. Essas elevadas prevalências na maioria das brássicas indicam a importância dessas doenças para as culturas nos estados, destacando a adaptabilidade dos patógenos aos ambientes e aos hospedeiros. Na média das diferentes espécies de brássicas, a alternariose e a podridão negra foram mais prevalentes em Pernambuco que em Santa Catarina (Tabela 1).

Os níveis de severidade da alternariose e da podridão negra foram baixos, tendo em vista que do total de 103 cultivos amostrados, $98 \%$ apresentou níveis de severidade das doenças inferiores a 5\%. Os níveis verificados assemelham-se aos constatados em cultivos convencionais de repolho nas safras 1997 e 1998 (Azevêdo et al., 2000), bem como na análise do comportamento de cultivares de brássicas no sistema orgânico (Rodrigues et al., 2004), ambos em Pernambuco. Principalmente no caso da alternariose, mesmo em níveis 
Tabela 1. Prevalência e severidade da alternariose e da podridão negra em cultivos orgânicos de brássicas nos estados de Pernambuco e Santa Catarina. Safra 2001/2002.

\begin{tabular}{|c|c|c|c|c|}
\hline \multirow{2}{*}{ Cultura } & \multicolumn{2}{|c|}{ Prevalência alternariose $(\%)^{1}$} & \multicolumn{2}{|c|}{ Prevalência podridão negra (\%) } \\
\hline & Pernambuco $^{2}$ & Santa Catarina $^{3}$ & Pernambuco & Santa Catarina \\
\hline Brócolis & 100 & 86,7 & 80,0 & 100 \\
\hline Couve-chinesa & 83,3 & 0,0 & 66,7 & 0,0 \\
\hline Couve-manteiga & 57,9 & 64,3 & 73,7 & 78,6 \\
\hline Couve-flor & 100 & 100 & 88,9 & 100 \\
\hline Repolho & 90,0 & 80,0 & 70,0 & 40,0 \\
\hline Média & 86,2 & 66,2 & 75,9 & 63,7 \\
\hline \multirow{2}{*}{ Cultura } & \multicolumn{2}{|c|}{ Severidade alternariose $(\%)^{4}$} & \multicolumn{2}{|c|}{ Severidade podridão negra $(\%)^{4}$} \\
\hline & Pernambuco $^{2}$ & Santa Catarina $^{3}$ & Pernambuco & Santa Catarina \\
\hline Brócolis & 1,87 aA5 & $1,25 \mathrm{aA}$ & $0,46 \mathrm{aB}$ & $1,48 \mathrm{aA}$ \\
\hline Couve-chinesa & $2,23 \mathrm{aA}$ & $0,00 \mathrm{aB}$ & $0,59 \mathrm{aA}$ & $0,00 \mathrm{bB}$ \\
\hline Couve-manteiga & $0,23 \mathrm{bA}$ & $0,15 \mathrm{aA}$ & 0,27 aA & $0,61 \mathrm{abA}$ \\
\hline Couve-flor & $1,29 a b A$ & $0,50 \mathrm{aA}$ & $0,53 \mathrm{aB}$ & $2,24 \mathrm{aA}$ \\
\hline Repolho & $1,46 \mathrm{aA}$ & $1,71 \mathrm{aA}$ & $1,12 \mathrm{aA}$ & $1,31 \mathrm{abA}$ \\
\hline Média & $1,42 \mathrm{~A}$ & $0,72 \mathrm{~A}$ & $0,59 \mathrm{~A}$ & $1,13 \mathrm{~A}$ \\
\hline
\end{tabular}

${ }^{1}$ Porcentagem de cultivos com a doença em relação ao total de cultivos amostrados; ${ }^{2}$ Considerando 20 cultivos de brócolis, 6 de couve-chinesa, 9 de couve-flor, 19 de couve-manteiga e 10 de repolho; ${ }^{3}$ Considerando 15 cultivos de brócolis, 3 de couve-chinesa, 2 de couve-flor, 14 de couve-manteiga e 5 de repolho; ${ }^{4}$ Estimada em cinco folhas basais por planta, pela porcentagem de área foliar lesionada com o auxílio de escalas diagramáticas (Azevêdo et al., 2000; Conn et al., 1990), em 50 plantas por cultivo; ${ }^{5}$ Médias seguidas pela mesma letra minúscula na coluna e maiúscula na linha, dentro de cada doença, não diferem significativamente entre si pelos testes de Kruskal-Wallis $(\mathrm{P}=0,05)$ e Wilcoxon $(\mathrm{P}=0,05)$, respectivamente.

baixos de severidade os prejuízos resultantes são importantes. Em couve-comum, brócolis e couve-flor as perdas devido à doença podem ser muito drásticas, pois atingem diretamente as partes comercializáveis, folhas no caso de couve-manteiga e inflorescências nos casos de brócolis e couve-flor, desqualificando os produtos. Em repolho e couve-chinesa é possível a eliminação das folhas doentes ("toilete"), mas ocorre a redução do rendimento e da remuneração dos produtos.

A severidade da alternariose variou significativamente entre as espécies de brássicas em Pernambuco, sendo o menor valor verificado em couve-manteiga, enquanto em Santa Catarina não foram constatadas diferenças entre as brássicas (Tabela 1). Variação significativa nos níveis de severidade da alternariose entre as espécies de brássicas também foi constatada por Rodrigues et al. (2004), ao analisarem o comportamento de cultivares no sistema orgânico, embora os maiores níveis tenham sido registrados em couve-flor. Em relação à podridão negra, inexistem outros estudos comparando a severidade em diferentes espécies de brássicas.
Quando comparados os dois estados em relação a uma mesma espécie de brássica, somente em couve-chinesa houve diferença significativa entre os níveis de severidade da alternariose, tendo em vista que em Santa Catarina não foi detectada a doença (Tabela 1). A não ocorrência da alternariose nos cultivos de couve-chinesa de Santa Catarina é surpreendente, tendo em vista que se encontravam freqüentemente ao lado de outras brássicas com sintomas da doença. Além disso, inexistem relatos de cultivares comerciais com elevados níveis de resistência à doença (Rodrigues et al., 2004), bem como de especialização fisiológica em A. brassicicola (Humpherson-Jones, 1992), embora tenha sido detectada variabilidade patogênica nas populações desse fungo (Michereff et al., 2003).

Em relação à podridão negra, somente em Santa Catarina houve diferença na severidade entre as brássicas, sendo registrados os menores níveis em couve-chinesa (Tabela 1). Na comparação dos estados, Pernambuco apresentou níveis de severidade significativamente superiores de podridão negra em couve-chinesa, enquanto Santa Catarina se destacou em brócolis e couve-flor (Tabela 2).

Quando consideradas as médias de severidade de cada doença no conjunto das brássicas, não foram constatadas diferenças significativas entre os dois estados (Tabela 1), embora nos períodos de realização dos levantamentos as condições climáticas nos dois estados tenham sido nitidamente distintas. Em Pernambuco, a temperatura média foi de $33,4^{\circ} \mathrm{C}$, com precipitação total de 53,1 mm, distribuída em 5,2 dias de chuva. Em Santa Catarina, a temperatura média foi de $23,7^{\circ} \mathrm{C}$, com precipitação total de 502,5 mm, distribuída em 38,3 dias de chuva.

O desenvolvimento das epidemias de alternariose e podridão negra em brássicas depende das condições ambientais, da resistência do hospedeiro e dos níveis de inóculo inicial. Embora as condições ambientais nos estados de Pernambuco e Santa Catarina tenham sido muito diferentes, principalmente em relação à elevada pluviosidade, que na maioria das vezes é correlacionada positivamente com a elevada severidade dessas doenças (Humpherson-Jones \& Phelps, 1989; Kocks \& Zadoks, 1996; Kocks et al., 1999; Verma \& Saharan, 1994), essa expectativa não se concretizou. Esses resultados indicam a grande adaptabilidade dos patógenos às diferentes condições ambientais, bem como a possibilidade de outros fatores terem sido determinantes para a ocorrência das doenças.

No caso da alternariose, a semelhança nos níveis de severidade entre os dois estados não chega a surpreender, tendo em vista que a flexibilidade ambiental de algumas espécies de Alternaria em relação à capacidade de causar doenças tem sido evidenciada em vários patossistemas (Rotem, 1994). No entanto, como X. campestris pv. campestris é disseminada principalmente durante a chuva (Williams, 1980), sendo o progresso da doença freqüentemente correlacionado com a freqüência de chuvas (Kocks \& Zadoks, 1996; Williams, 1980), seriam esperadas diferenças significativas na severidade da doença entre os dois estados. 
Tabela 2. Severidade da alternariose (AL) e da podridão negra (PN) em cultivos orgânicos de brócolis e couve-manteiga nos estados de Pernambuco e Santa Catarina, considerando local de cultivo, cultivar, número de plantas e idade das plantas. Safra 2001/2002.

\begin{tabular}{|c|c|c|c|c|c|}
\hline \multicolumn{3}{|c|}{ Severidade Pernambuco $(\%)^{1}$} & \multicolumn{3}{|c|}{ Severidade Santa Catarina(\%) ${ }^{1}$} \\
\hline \multicolumn{6}{|c|}{ Brócolis } \\
\hline Local & $\mathrm{AL}$ & PN & Local & $\mathrm{AL}$ & PN \\
\hline Bom Jardim & $0,06 a^{2}$ & $0,07 a$ & Araranguá & $1,20 \mathrm{~b}$ & $1,44 \mathrm{a}$ \\
\hline Chã Grande & $1,92 \mathrm{a}$ & $1,00 \mathrm{a}$ & Biguaçú & $0,27 \mathrm{~b}$ & $1,40 \mathrm{a}$ \\
\hline Gravatá & $2,28 a$ & $0,40 \mathrm{a}$ & Florianópolis & $0,00 \mathrm{~b}$ & $0,32 \mathrm{a}$ \\
\hline \multirow[t]{4}{*}{ Glória de Goitá } & $0,02 \mathrm{a}$ & $0,00 \mathrm{a}$ & Paulo Lopes & $0,95 \mathrm{~b}$ & 3,81 a \\
\hline & & & Sta. Rosa Lima & $1,39 b$ & $2,04 \mathrm{a}$ \\
\hline & & & Sto. Amaro Imperatriz & $0,02 b$ & $1,58 \mathrm{a}$ \\
\hline & & & Urubici & $4,11 \mathrm{a}$ & $0,07 \mathrm{a}$ \\
\hline Cultivar & $\mathbf{A L}$ & PN & Cultivar & $\mathbf{A L}$ & PN \\
\hline Precoce de Piracicaba & $2,57 \mathrm{a}$ & $0,20 \mathrm{a}$ & Legacy & $4,11 \mathrm{a}$ & $0,07 a$ \\
\hline \multirow[t]{3}{*}{ Piracicaba de Verão } & $1,03 \mathrm{~b}$ & 0,79 a & Marathon & $0,49 b$ & $1,32 \mathrm{a}$ \\
\hline & & & Precoce de Piracicaba & $0,68 \mathrm{~b}$ & $0,77 \mathrm{a}$ \\
\hline & & & Piracicaba de Verão & $1,13 b$ & $2,32 \mathrm{a}$ \\
\hline Número de plantas & $\mathrm{AL}$ & PN & Número de plantas & $\mathbf{A L}$ & PN \\
\hline $50-150$ & $1,50 \mathrm{a}$ & $0,68 \mathrm{a}$ & $50-500$ & $0,83 a$ & $1,82 a$ \\
\hline $151-300$ & $2,68 a$ & $0,33 a$ & $501-1000$ & $0,00 \mathrm{a}$ & $0,06 \mathrm{a}$ \\
\hline$>300$ & $1,32 a$ & $0,22 a$ & $>1000$ & $2,62 \mathrm{a}$ & $0,99 a$ \\
\hline Idade das plantas & $\mathrm{AL}$ & PN & Idade das plantas & $\mathrm{AL}$ & PN \\
\hline $1-50$ & $0,37 \mathrm{~b}$ & $0,20 \mathrm{a}$ & $1-50$ & $0,65 \mathrm{a}$ & $0,60 a$ \\
\hline $51-100$ & $2,47 a$ & $0,33 a$ & $51-100$ & $1,46 a$ & $1,66 \mathrm{a}$ \\
\hline$>100$ & $0,74 \mathrm{~b}$ & $1,92 \mathrm{a}$ & $>100$ & $1,13 \mathrm{a}$ & $1,91 \mathrm{a}$ \\
\hline \multicolumn{6}{|c|}{ Couve-manteiga } \\
\hline Local & $\mathrm{AL}$ & PN & Local & $\mathrm{AL}$ & PN \\
\hline Bom Jardim & $0,21 \mathrm{a}$ & $0,30 \mathrm{a}$ & Araranguá & $0,01 \mathrm{a}$ & $0,34 \mathrm{~b}$ \\
\hline Chã Grande & $0,03 a$ & $0,09 a$ & Biguaçú & $0,00 \mathrm{a}$ & $0,10 \mathrm{~b}$ \\
\hline Gravatá & $0,54 \mathrm{a}$ & $0,51 \mathrm{a}$ & Florianópolis & $0,00 \mathrm{a}$ & $0,00 \mathrm{~b}$ \\
\hline \multirow[t]{4}{*}{ Glória de Goitá } & $0,02 a$ & $0,00 \mathrm{a}$ & Paulo Lopes & $0,00 \mathrm{a}$ & $0,95 \mathrm{~b}$ \\
\hline & & & Sta. Rosa & $0,28 a$ & $0,46 \mathrm{~b}$ \\
\hline & & & Sto. Amaro & $0,00 \mathrm{a}$ & $3,50 \mathrm{a}$ \\
\hline & & & Urubici & $0,06 \mathrm{a}$ & $0,12 b$ \\
\hline Número de plantas & $\mathrm{AL}$ & PN & Número de plantas & $\mathbf{A L}$ & PN \\
\hline $50-150$ & $0,20 \mathrm{a}$ & $0,33 a$ & $50-150$ & $0,26 \mathrm{a}$ & $0,59 a$ \\
\hline $151-300$ & $0,02 a$ & $0,00 \mathrm{a}$ & $151-300$ & $0,03 a$ & $0,05 a$ \\
\hline$>300$ & $0,35 a$ & $0,19 a$ & $>300$ & $0,03 a$ & $0,87 a$ \\
\hline Idade das plantas & $\mathrm{AL}$ & PN & Idade das plantas & $\mathrm{AL}$ & PN \\
\hline $1-100$ & $0,29 a$ & $0,16 a$ & $1-100$ & $0,02 a$ & $0,68 a$ \\
\hline $101-200$ & $0,18 a$ & $0,41 \mathrm{a}$ & $101-200$ & $0,24 \mathrm{a}$ & $0,66 \mathrm{a}$ \\
\hline$>200$ & $0,01 \mathrm{a}$ & $0,38 a$ & $>200$ & $0,12 a$ & $0,58 a$ \\
\hline
\end{tabular}

Nos dois estados, os locais de cultivo de brócolis não diferiram significativamente entre si quanto aos níveis de severidade da podridão negra. Em relação à alternariose, os cultivos em Urubici diferiram significativamente dos demais de Santa Catarina, enquanto não houve diferença entre os locais de cultivo em Pernambuco (Tabela 2).

Nos plantios de brócolis de Pernambuco a cultivar Piracicaba de Verão apresentou severidade da alternariose significativamente inferior ao verificado na cultivar Precoce de Piracicaba, embora sem diferir desta em relação à podridão negra (Tabela 2). Nos plantios de brócolis de Santa Catarina foram constatadas diferenças significativas entre as cultivares somente em relação à severidade da alternariose, sendo que 'Legacy' apresentou nível significativamente superior às demais cultivares (Tabela 2), assemelhando-se ao constatado previamente quando essa cultivar foi confrontada com outras para avaliação dos níveis de resistência à doença no sistema orgânico (Rodrigues et al., 2004).

Quando comparados os números de plantas de brócolis por cultivo, não foram constatadas diferenças significativas entre as classes quanto aos níveis de severidade das doenças nos dois estados (Tabela 2). Em relação à idade das plantas de brócolis, em Santa Catarina não foram constatadas diferenças signi- 
ficativas entre as classes quanto aos níveis de severidade das doenças. Em Pernambuco foram verificadas diferenças significativas na severidade da alternariose entre as classes de idade, sendo superior na classe de 51-100 dias de idade (Tabela 2).

Nos dois estados os locais de cultivo de couve-manteiga não diferiram significativamente entre si quanto aos níveis de severidade da alternariose. Quanto à podridão negra, somente em Santa Catarina foram constatadas diferenças entre os locais de cultivo, sendo que em Santo Amaro da Imperatriz foram registrados os maiores níveis da doença (Tabela 2). Em todas as áreas amostradas, nos dois estados, a couvemanteiga era propagada vegetativamente, por clones tradicionais não identificados, sem a utilização de cultivares propagados por sementes.

Não foram constatadas diferenças significativas entre as classes de números de plantas e idades da couve-manteiga quanto aos níveis de severidade da alternariose e da podridão negra (Tabela 2).

As variações significativas nos níveis de severidade da alternariose e da podridão negra em algumas das situações consideradas nos cultivos de brócolis e couve-manteiga nos dois estados podem estar associadas à presença de maior quantidade de inóculo na época de plantio ou chegada de inóculo externo durante o ciclo da cultura, a exemplo do verificado em Pernambuco quando analisadas epidemias dessas doenças em cultivos de repolho no sistema convencional (Azevedo et al., 2002).

Em relação às espécies de Alternaria causadoras de alternariose, em Pernambuco houve predominância de $A$. brassicicola, detectada em $69 \%$ das amostras avaliadas, enquanto $A$. brassicae foi constatada em $31 \%$ das amostras, sendo as duas espécies constatadas freqüentemente sob couve-chinesa, couve-flor, couve-manteiga e repolho. Em brócolis, A. brassicae foi registrada em apenas uma área de cultivo, o que indica uma predominância de A. brassicicola. A predominância de $A$. brassicicola em Pernambuco confirma resultados anteriores (Azevedo et al., 2000; Michereff et al., 2003; Rodrigues et al., 2004), tendo em vista a maior esporulação e infecção desse patógeno em temperaturas elevadas e adaptação às interrupções do período de molhamento (Verma \& Saharan, 1994). Por outro lado, A. brassicae não havia sido registrada previamente em cultivos de brássicas nesse estado.

Em Santa Catarina foi constatada somente A. brassicicola, infectando brócolis, couve-comum, couve-flor e repolho, mas não couve-chinesa. Esse resultado foi inesperado, tendo em vista que as condições climáticas predominantes foram mais favoráveis à $A$. brassicae (Verma \& Saharan, 1994).

As intensidades das doenças no cultivo orgânico verificados nesse estudo corroboram com os resultados de Rodrigues et al. (2004), demonstrando que o suposto equilíbrio nutricional dos cultivos orgânicos não é suficiente para eliminar ou mesmo reduzir a severidade dos sintomas das doenças. Uma hipótese a considerar é que a intensidade das doenças nos plantios orgânicos pode estar associada ao estresse nutricional a que as plantas foram submetidas, tendo em vista que as cultivares utilizadas foram selecionadas originalmente para produção em sistema convencional, onde são utilizados principalmente fertilizantes minerais solúveis com imediata disponibilidade de nutrientes para as plantas, diferentemente do que acontece no sistema orgânico, onde predomina a utilização de fertilizantes orgânicos, que apresentam lenta liberação de nutrientes para absorção vegetal (Kiehl, 1993). Nesse contexto, a falta de germoplasmas adaptadas aos sistemas orgânicos de produção tem sido destacada como uma das sérias limitações para o manejo de doenças em hortaliças e outras culturas (Bueren, 2002). Além da utilização de cultivares resistentes, a destruição de restos culturais e a rotação de culturas devem ser aplicadas para o manejo da alternariose e podridão negra das brássicas.

Ficou evidente a importância da alternariose e a podridão negra nos cultivos orgânicos de brássicas em Pernambuco e Santa Catarina. As informações obtidas nos levantamentos, principalmente com as diferentes comparações efetuadas, subsidiarão e elaboração de novos estudos sobre epidemiologia e estratégias de manejo da alternariose e da podridão negra nos dois estados, visando minimizar as perdas na produção orgânica de brássicas.

\section{REFERÊNCIAS}

AZEVÊDO SS; MARIANO RLR; MICHEREFF SJ. 2002. Epidemiologia comparativa da podridão negra e da alternariose do repolho no Agreste de Pernambuco. Fitopatologia Brasileira 27: 17-26.

AZEVÊDO SS; MICHEREFF SJ; MARIANO RLR. 2000. Levantamento da intensidade da podridão negra e da alternariose do repolho no Agreste de Pernambuco e determinação do tamanho das amostras para quantificação dessas doenças. Summa Phytopathologica 26: 299-306.

Bueren TL. Organic plant breeding and propagation: concepts and strategies. 2002. Thesis (PhD in Plant Breeding)-Wageningen Agricultural University, Wageningen, The Netherlands. 209p.

CAMPBELL CL; MADDEN LV. 1990. Introduction to plant disease epidemiology. New York: John Wiley. 532p.

CONN KL; TEWARI JP; AWASTHI RP. 1990. A disease assessment key for Alternaria blackspot in rapeseed and mustard. Canadian Plant Disease Survey 70: 19-22.

HUMPHERSON-JONES FM. 1992. Epidemiology and control of dark leaf spot of brassicas. In: CHELKOWSKI J; VISCONTI A (eds). Alternaria: biology, plant diseases and metabolites. Amsterdam: Elsevier. p. 267-288.

HUMPHERSON-JONES FM; PHELPS K.1989. Climatic factors influencing spore production in Alternaria brassicae and Alternaria brassicicola. Annals of Applied Biology 114: 449-459.

KIEHL EJ. 1993. Fertilizantes organominerais. Piracicaba: Agronômica Ceres. 189p.

KING JE. 1980. Cereal survey methodology in England and Wales. In: TENG, P.S.; KRUPA, S.V. (eds). Crop loss assessment which constrain production and crop improvement in agriculture and forestry. Minnesota: University of Minnesota. p. 124-133. (Agricultural Experiment Station. Miscellaneous Publication, 7).

KOCKS CG; ZADOKS JC. 1996. Cabbage refuse piles as sources of inoculum for black rot epidemics. Plant Disease 80: 789-792.

KOCKS CG; ZADOKS JC; RUISSEN TA. 1999. Spatio-temporal development of black rot (Xanthomonas campestris pv. campestris) in cabbage in relation to initial inoculum levels in field plots in The Netherlands. Plant Pathology 48: 176-188.

MARINGONI AC. 1997. Doenças das crucíferas (brócolis, couve, couve-chinesa, couve-flor, rabanete, repolho e rúcula). In: KIMATI $\mathrm{H}$; AMORIM L; BERGAMIN FILHO A; CAMARGO LEA; REZENDE JAM (eds). Manual de fitopatologia: doenças das plantas cultivadas. 3. ed. São Paulo: Agronômica Ceres 2: 315-324. 
MICHEREFF SJ; NORONHA MA; ROCHA JÚNIOR OM; SILVA JA; MIZUBUTI ESG

2003. Variabilidade de isolados de Alternaria brassicicola no estado de Pernambuco. Fitopatologia Brasileira 28: 656-663.

RAMSEY GB; SMITH MA. 1961. Market diseases of cabbage, cauliflower, turnips, cucumbers, melons and related crops. Washington: United States Department of Agriculture. 35p.
RODRIGUES VJLB; MICHEREFF SJ; MENEZES D; AGUIAR FILHO MR; SILVA LGC; BIONDI CM. 2004. Epidemiologia comparativa da alternariose em cultivares de brássicas sob cultivo convencional e orgânico. Summa Phytopathologica 30: 226-233.

ROTEM J. 1994. The genus Alternaria: biology, epidemiology and pathogenicity. St. Paul: APS Press. 326p.
VAN BRUGGEN A. 2001. Switching over to organic farming systems: consequences for plant pathological research. Summa Phytopathologica 27: 145.

VERMA PR; SAHARAN GS. 1994. Monograph on Alternaria diseases of crucifers. Saskatoon: Minister of Supply and Services Canada. 162p.

WILLIAMS PH. 1980. Blackrot: a countinuing threat to world crucifers. Plant Disease 64: 736-742. 